\title{
Enhanced Mobility and Large Linear Nonsaturating Magnetoresistance in the Magnetically Ordered States of $\mathrm{TmNiC}_{2}$
}

\author{
Kamil K. Kolincio $\odot,{ }^{1}$ Marta Roman $\odot,{ }^{1}$ and Tomasz Klimczuk $\oplus^{1,2}$ \\ ${ }^{1}$ Faculty of Applied Physics and Mathematics, Gdansk University of Technology, Narutowicza 11/12, 80-233 Gdansk, Poland \\ ${ }^{2}$ Advanced Materials Centre, Gdansk University of Technology, ul. Narutowicza 11/12, 80-233 Gdansk, Poland
}

(Received 20 June 2020; revised 2 September 2020; accepted 11 September 2020; published 22 October 2020)

\begin{abstract}
We have studied the magnetic, magnetotransport, and galvanomagnetic properties of $\mathrm{TmNiC}_{2}$. We find that the antiferromagnetic and field induced metamagnetic and ferromagnetic orderings do not suppress the charge density wave. The persistence of Fermi surface pockets, open as a result of imperfect nesting accompanying the Peierls transition, results in an electronic carriers mobility of the order of $4 \times$ $10^{3} \mathrm{~cm}^{2} \mathrm{~V}^{-1} \mathrm{~s}^{-1}$ in ferromagnetic state, without any signatures for a significant deterioration of nesting properties. This is independently evidenced by high, nonsaturating linear magnetoresistance reaching $440 \%$ at $T=2 \mathrm{~K}$ and an analysis of the Hall conductivity. We thus demonstrate that, the coexistence of charge density wave and magnetism provides an alternative route to maintain high electronic mobility in the magnetically ordered state.
\end{abstract}

DOI: 10.1103/PhysRevLett.125.176601

Recent years have witnessed the search for materials which exhibit both high mobility of electronic carriers and magnetic ordering. This interest originates from their potential applications in spintronic devices [1,2]. Despite continuous efforts, the number of such systems is limited mostly to materials hosting topological Dirac or Weyl states [3-7], or other sharp peculiarities of the electronic structure [8]. Besides the topological states, magnetism is not an ally of the increased mobility, as it introduces additional spin disorder or spin dependent scattering, which predominantly leads to the increase of the electronic effective mass [9]. Another natural precursor for enhanced mobility is the Peierls transition leading to formation of the charge density wave $(\mathrm{CDW})[10,11]$. This type of electronic ordering is accompanied with the Fermi surface (FS) nesting which additionally might open small FS pockets containing high mobility carriers resulting in strong transport and thermoelectric responses [12-14]. However, the Peierls instability typically competes with magnetic order [15-18]. One of the rather rare systems in which CDW coexists with magnetism is the $R \mathrm{NiC}_{2}$ family with $R$ being a rare earth metal. The CDW in early lanthanide based $R \mathrm{NiC}_{2}$ compounds is characterized by the $q_{1}=(0.5 ; 0.5+\delta ; 0)$ vector, which for heavier $R$ evolves to a triply commensurate $q_{2}=$ $(0.5 ; 0.5 ; 0.5)$ [19-22]. The former type of modulation was previously found to be mutually related with

Published by the American Physical Society under the terms of the Creative Commons Attribution 4.0 International license. Further distribution of this work must maintain attribution to the author(s) and the published article's title, journal citation, and DOI. magnetism. On the one hand, CDW nesting was proposed to have a constructive impact on the antiferromagnetic (AFM) state, which in $\mathrm{GdNiC}_{2}$ and $\mathrm{NdNiC}_{2}$ is represented by a propagation vector coincident with $q_{1}$ [23-25]. This positive influence was proposed [19,20,26-28] to be caused by Ruderman-Kittel-Kasuya-Yosida (RKKY) [29-31] mechanism mediating interactions between local magnetic moments through conduction electrons. On the other hand, the magnetism has a rather destructive impact on CDW: while AFM partially weakens the charge density wave state [20,32-34], the ferromagnetic (FM) or metamagnetic (MM) transitions suppress it entirely [32-36]. The latter type of CDW modulation not only was found to be robust against antiferromagnetic transition as in (Dy-Er) $\mathrm{NiC}_{2}$ [22], but also to inflict strong Fermi surface nesting, concomitant with opening of high mobility pockets in nonmagnetic $\mathrm{YNiC}_{2}$ [37]. This context motivated us to study the magnetotransport properties of $\mathrm{TmNiC}_{2}$, which shows both CDW at $T_{P}=394 \mathrm{~K}$ [38] and antiferromagnetism at $T_{N} \simeq 5 \mathrm{~K}[23,39-41]$. Phase diagram is enriched by field induced metamagnetic and ferromagnetic crossovers occurring at $\mu_{0} H_{c 1} \simeq 0.9 \mathrm{~T}$, and $\mu_{0} H_{c 2} \simeq 1.2 \mathrm{~T}$, respectively [39]. Coexistence of these entities combines the physical properties associated with them, predisposing heavy lanthanide $\mathrm{TmNiC}_{2}$ to exhibit high values of electronic mobility in magnetically ordered state.

The polycrystalline sample of $\mathrm{TmNiC}_{2}$ was synthesized via the arc-melting method followed by thermal annealing to ensure an adequate homogeneity and quality. We have previously described the details of synthesis process in Ref. [33]. Physical properties were measured by a Quantum Design Physical Properties Measurement System (PPMS). The AC Measurement System (ACMS) option was used to 

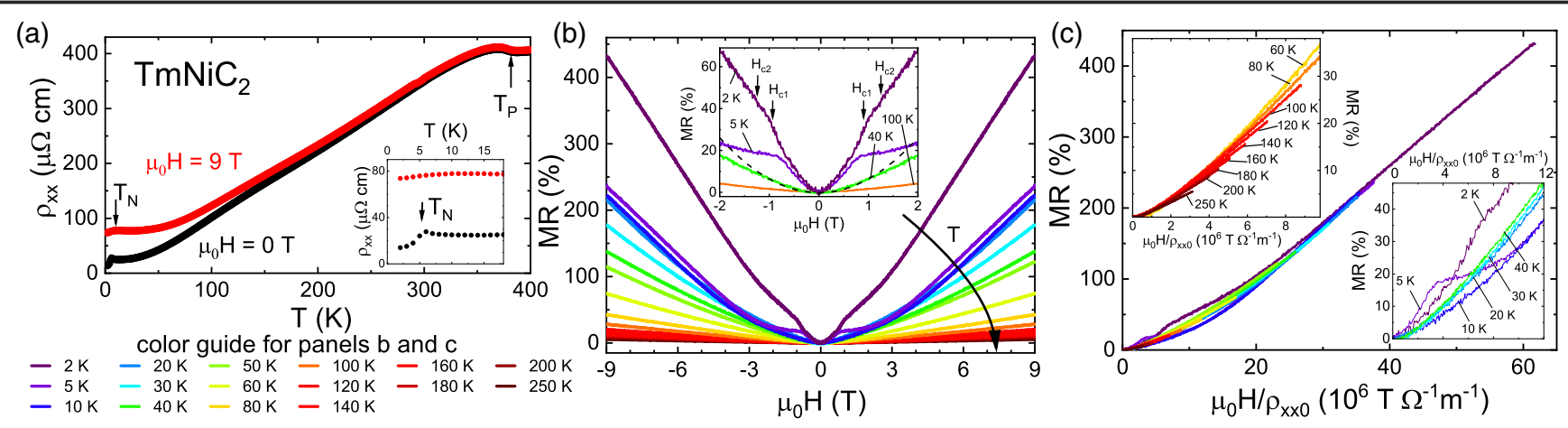

FIG. 1. (a) Thermal dependence of $\rho_{x x}$ at zero field and $\mu_{0} H=9 \mathrm{~T}$, inset shows an expanded view of the low temperature range. (b) Magnetic field dependence of MR. Dashed line is an exemplary fit to the low field data with Eq. (5). (c) Kohler's plot of MR, the insets depict the violation of the Kohler's rule at moderate temperatures (upper), and in the vicinity of $T_{N}$ (lower).

measure the magnetic properties. Transport measurements were conducted with a standard four-probe method with thin Pt wires spark welded to the polished sample surface. The magnetic field for magnetoresistance and Hall measurements was applied perpendicularly to the sample surface, and thus to the current direction. The off-diagonal resistivity was measured with reversing of the magnetic field and carefully antisymmetrized in order to eliminate the spurious longitudinal contribution from possible misalignment of electrical contacts. The magnetization measurements performed with our sample (see the Supplemental Material [42] for details) are in agreement with the previous reports $[23,39,41]$.

Figure 1(a) shows the thermal dependence of resistivity $\rho_{x x}$ measured both in the zero-field condition and with applied $\mu_{0} H=9 \mathrm{~T}$ magnetic field. The zero field curve was reported by us previously in Ref. [38] where we evidenced the Peierls instability at $T_{P}$ manifested as a metal-metal transition, characteristic for quasi-2D material with imperfect FS nesting. Here we extend the transport study by analysis of the resistivity measured under applied magnetic field, and also in order to explore the interplay between CDW and magnetism, we put an additional focus to the vicinity of the AFM transition, which is accompanied by a reduction of resistivity. The relative drop of resistivity at magnetic ordering temperature $[\rho(6 K-\rho(2 K)) / \rho(6 K)] \simeq 0.43$ is one of the lowest amongst magnetic $\mathrm{RNiC}_{2}$. In particular, the corresponding ratios for $\mathrm{GdNiC}_{2}$ and $\mathrm{NdNiC}_{2}$ in which CDW state was partially suppressed by AFM transition, yield 0.5 and 0.72 , respectively $[20,26,32]$ or 0.9 in $\mathrm{SmNiC}_{2}$ where the charge density wave is completely obliterated in the FM state [36]. This comparison delivers a first suggestion that in $\mathrm{TmNiC}_{2}$ the Peierls instability is weakly influenced, or even intact at $T_{N}$ and the relatively low resistivity drop stems mostly from the quenching of spin disorder and thus of the underlying scattering term. The application of external magnetic field significantly increases the resistance in the CDW state and the impact of $\mu_{0} H$ grows as temperature is lowered. To obtain a more detailed picture, we have followed the magnetic field dependence of magnetoresistance defined as:

$$
\mathrm{MR}=\frac{\rho_{x x}\left(\mu_{0} H\right)-\rho_{x x}\left(\mu_{0} H=0\right)}{\rho_{x x}\left(\mu_{0} H=0\right)} \times 100 \% .
$$

The $\operatorname{MR}\left(\mu_{0} H\right)$ curves displayed in Fig. 1(b) show a monotonical increase with magnetic field. In the paramagnetic (PM) state, the magnetoresistance shows a classical, parabolic dependence on applied $\mu_{0} H$, which evolves to a linear behavior in the high field limit. There are two models commonly used to explain the origin of linear magnetoresistance: classical Parish-Littlewood one, describing strongly disordered material $[44,45]$ or Abrikosov's quantum approach applicable beyond the quantum critical limit [46-48], however neither of them seems to be relevant here due to a lack of signatures of a substantial disorder and a rather large carrier concentration, respectively. In CDW metal the linear magnetoresistance, apart from these two models, can stem as well from the order parameter fluctuations $[49,50]$ or curved trajectories covered by the carriers in the partially nested FS [51]. One cannot however exclude the most simplistic scenario, in which the linear MR is a product of a mixed signal from the saturating and not-saturating components stemming from closed and open FS orbits, respectively [52,53]. MR consequently increases as temperature is lowered, reaching $\simeq 440 \%$ at $T=2 \mathrm{~K}$ and $\mu_{0} H=9 \mathrm{~T}$ without any signatures of saturation. Both the linear dependence on $\mu_{0} H$ and magnitude of $\mathrm{MR}$ in $\mathrm{TmNiC}_{2}$ is similar to its nonmagnetic analog $\mathrm{YNiC}_{2}$ [37]. The main difference is observed in the low field limit: in $\mathrm{TmNiC}_{2}$, in the vicinity of $T_{N}$, the low field MR shows a more complex character with local inflections, likely corresponding to the field induced MM and FM transformations [39], yet even within the ferromagnetic dome, the high field MR retains the character and magnitude from the PM state. This behavior is entirely different of what previously was observed in $\mathrm{GdNiC}_{2}, \mathrm{NdNiC}_{2}$, or $\mathrm{SmNiC}_{2}$, where charge density wave is completely destructed by field induced ferromagnetic or metamagnetic transitions [32-36]. A consequence of CDW suppression is an avalanche decrease of resistivity when the CDW gap disappears, which is not observed at the magnetic crossovers in $\mathrm{TmNiC}_{2}$. 

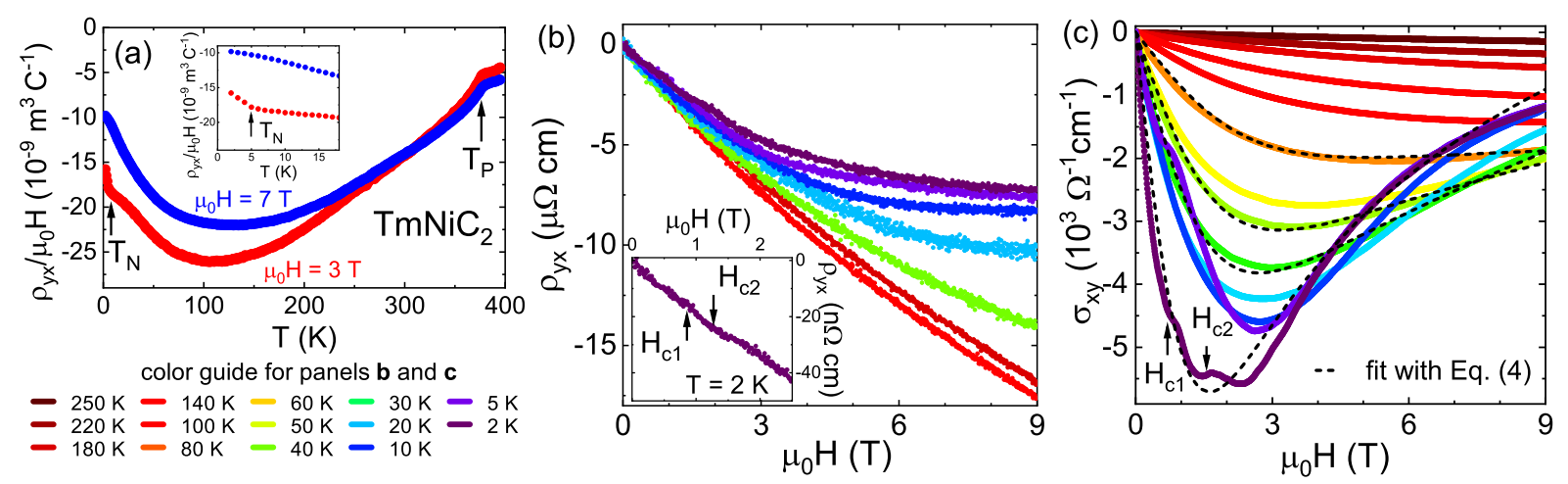

FIG. 2. (a) Thermal dependence of $\rho_{y x} /\left(\mu_{0} H\right)$ measured at two different fields. Inset shows an expanded view of the low temperature range. (b) $\rho_{y x}\left(\mu_{0} H\right)$ for selected temperatures. Inset shows the expanded view for the low field limit of $T=2 \mathrm{~K}$ curve. (c) $\rho_{y x}$ as a function of $\mu_{0} H$. Black dashed lines are typical fits with Eq. (4).

A complementary tool allowing the further discussion of magnetoresistance is delivered by the Kohler's scaling. This approach predicts [54,55], that in case of field independent scattering, uniform over the Fermi surface, and only a single type of carriers with constant concentration participating in electronic transport, all the plots:

$$
\mathrm{MR}=f\left(\omega_{c} \tau\right)=f\left(\frac{\mu_{0} H}{\rho_{x x}\left(\mu_{0} H=0\right)}\right)
$$

shall collapse to a single curve ( $\tau$ and $\omega_{c}$ are relaxation time and cyclotron frequency, respectively). Typically, this scaling is not used in magnetic systems, where scattering becomes field dependent, leading to the violation of the Kohler's rule. In $\mathrm{TmNiC}_{2}$ however, the low $T_{N}$ and large magnetoresistance in the paramagnetic state allows us to neglect the impact of magnetic scattering at higher temperatures, which thus opens a window for at least a qualitative discussion.

As seen in the Fig. 1(c), the Kohler's plots roughly follow a single trend line indicating the dominance of a positive term in the whole temperature range. Small, yet significant deviations of different kinds are observed in the high and low temperature limits, respectively. As depicted in the upper inset of Fig. 1(c), at temperatures far above $T_{N}$, the scaling plots do not overlap. The subsequent curves consequently turn higher as $T$ is decreased. An adverse deviation direction is observed at $T<50 \mathrm{~K}$, where the curves initially pressed below the main trend line start to overlap for higher fields. The strongest violation of the Kohler's rule is observed below $T_{N}$ [lower inset of Fig. 2(c)], where the curves for $T=2$ and $5 \mathrm{~K}$ first show an upturn, yet at a higher field they become parallel to the main trend line. The different directions of these violations allow us to identify and compare the underlying mechanisms. In the high temperature limit, they can stem from both reconstruction of the Fermi surface driven by the $\mathrm{CDW}$ or the presence of more than one type of carriers from the pockets in the unnested residue of FS. This latter scenario seems more relevant at temperature below $T_{P} / 2$ where FS modification is expected to be already completed. The violations seen in the vicinity and below $T_{N}$, where the magnetic fluctuations are to be considered, likely stem from the magnetic field dependent scattering. Above $T_{N}$ the spin disorder is slightly decreased, which accounts for the downturn of the corresponding plots, or increased upon the transformation from AFM to MM and FM state, in which not all the spins are perfectly arranged up to $\mu_{0} H=9 \mathrm{~T}$. Despite of these features, the strong positive MR appearing in the CDW state remains a dominant term also below $T_{N}$. This indicates that the prevailing mechanism of magnetoresistance in $\mathrm{TmNiC}_{2}$ remains unchanged across the magnetic crossovers from paramagnetic towards ferromagnetic state.

As the emergence of strong magnetoresistance is already a hallmark of high mobility, a more detailed picture can be obtained by the analysis of the off-diagonal components of resistivity and conductivity. An additional advantage of the exploration of the Hall effect is that it provides a direct insight into the concentration of electronic carriers. Since the consequence of FS nesting (induced by Peierls transition) is a reduction of the carrier concentration, the analysis of the Hall effect allows us to explore the evolution of charge density wave even when the diffraction experiment in the search for satellite reflections cannot be performed. In the previous report [38], by the analysis of the temperature dependence of Hall resistivity $\rho_{y x}$, we have evidenced the carrier condensation accompanying the Peierls transition in polycrystalline $\mathrm{TmNiC}_{2}$. Here, we extend the study of the Hall effect, and examine the offdiagonal components of both resistivity and conductivity at various fields and temperatures in order both to follow the evolution of CDW and the mobility of conduction carriers on the path between the Peierls transition to magnetically ordered state. The thermal dependence of Hall resistivity divided by magnetic field $\rho_{y x} /\left(\mu_{0} H\right)$ is presented in 
Fig. 2(a). As reported previously [38], curves descent at $T_{P}$, due to the partial FS gap opening and reduction of electronic density, which is followed by a minimum and upturn at lower $T$. Here we observe that $\rho_{y x}$ becomes nonlinear with field, which is depicted in Fig. 2(b). This likely originates from a partial compensation due to the presence of more than one type of electronic carriers, which will account for large magnetoresistance as seen in nonmagnetic $\mathrm{YNiC}_{2}$ and $\mathrm{LuNiC}_{2}$. In magnetic $\mathrm{TmNiC}_{2}$ this behavior might also partially stem from the anomalous Hall component [56] as observed in other $R \mathrm{NiC}_{2}$ [32-34]. In the context of the interplay between CDW and magnetism, it is useful to track the $\rho_{y x} /\left(\mu_{0} H\right)$ across the metamagnetic and ferromagnetic transformations. The inset of Fig. 2(b) shows the expanded view for the curve measured at $T=2 \mathrm{~K}$. The insignificant response of $\rho_{y x} /\left(\mu_{0} H\right)$ at $H_{c 1}$ or $H_{c 2}$ stands in contrast to $\mathrm{GdNiC}_{2}$ [32] and $\mathrm{NdNiC}_{2}$ [34], where an abrupt upturn of Hall resistivity is seen when previously condensed carriers are released as CDW is suppressed by the MM and FM transitions. The modification of carriers concentration at these points appears to be negligible in $\mathrm{TmNiC}_{2}$.

For an insight into the carrier mobilities and concentrations, we calculate the Hall conductivity $\sigma_{x y}$ :

$$
\sigma_{x y}\left(\mu_{0} H\right)=\frac{\rho_{y x}}{\rho_{y x}^{2}+\rho_{x x}^{2}} .
$$

The magnetic field dependence of $\sigma_{x y}$ is depicted in Fig. 2(c). In the high temperature limit, Hall conductivity is a decreasing function of magnetic field. At lower $T$, the curves develop into a nonmonotonous character with pronounced minimum, which gradually shifts towards lower magnetic field at lower temperatures.

In paramagnetic materials, the inverse of a magnetic field corresponding to an extremum of the Hall conductivity $\sigma_{x y}$, is a direct measure of the dominant electronic mobility $\mu_{\text {ext }}$ [57,58]. Typically, in high mobility magnetic systems, the anomalous component of the Hall effect is neglected and $\mu$ is successfully determined $[8,59,60]$ as it is deemed that the ordinary component prevails over the anomalous one. As it has been observed previously [32-34], the impact of anomalous Hall effect in magnetic $R \mathrm{NiC}_{2}$ is rather strong and cannot be neglected. Then, to provide a more precise estimate of mobility, we have adopted (with a small modification) the simplified two-band model $[61,62]$ :

$\sigma_{x y}\left(\mu_{0} H\right)=n_{H} e \mu_{H}^{2} \mu_{0} H\left(\frac{1}{1+\mu_{H}^{2}\left(\mu_{0} H\right)^{2}}+C\right)+S_{A} M$.

This approach assumes one high mobility band, with concentration $n_{H}$ and mobility $\mu_{H}$ dominating in the low temperature limit, while other band(s) with much smaller $\mu$ contribute to the parameter $C$. This method was successfully used for paramagnetic $\mathrm{YNiC}_{2}$ and $\mathrm{LuNiC}_{2}$ [37] and to make it applicable also for magnetic $\mathrm{TmNiC}_{2}$, we have added the anomalous component $S_{A} M$, proportional to magnetization. Even despite the simplified character of the used model, the number of derived parameters remains relatively large. For that reason, to constrain the fit we have assumed constant anomalous Hall coefficient $S_{A}=7.6 \times 10^{3} \mu \Omega^{-1} \mathrm{~cm}^{-1}$ per $\mu_{B} / f . u$. determined from a free fit at $T=30 \mathrm{~K}$. As it can be seen in Fig. 2(c), our approach provides a well satisfactory description of the experimental data ( $C$ parameter is at the expected order of $\left.10^{-2}-10^{-3}\right)$. A larger, yet not significant discrepancy is observed at lowest temperature, as the Eq. (4) does not capture the field dependent renormalization of the scattering processes under applied magnetic field. This drawback naturally results in enlarged uncertainty, yet considering the dominance of quadratic and linear MR over the impact of magnetic scattering, we can conclude that the used approach provides a reasonable estimation of $\mu$.

A complementary, albeit less definitive, method used for estimating the mobility is provided directly from the magnetoresistance. In the low field limit, where it follows the quadratic dependence on field, the average mobility is estimated from the fits with semiclassical formula:

$$
\mathrm{MR}=\mu_{\mathrm{MR}}^{2}\left(\mu_{0} H\right)^{2} .
$$

The reliable fits are however obtained only for curves at $T \geq 20 \mathrm{~K}$, where the spin scattering does not distort the $\left(\mu_{0} H\right)^{2}$ character of low field magnetoresistance.

The results of these three approaches are summarized in Fig. 3. As illustrated in Fig. 3(a), the concentration of the minority carriers $n_{H}$ increases as temperature is decreased, which is concomitant with the decrease of the number of

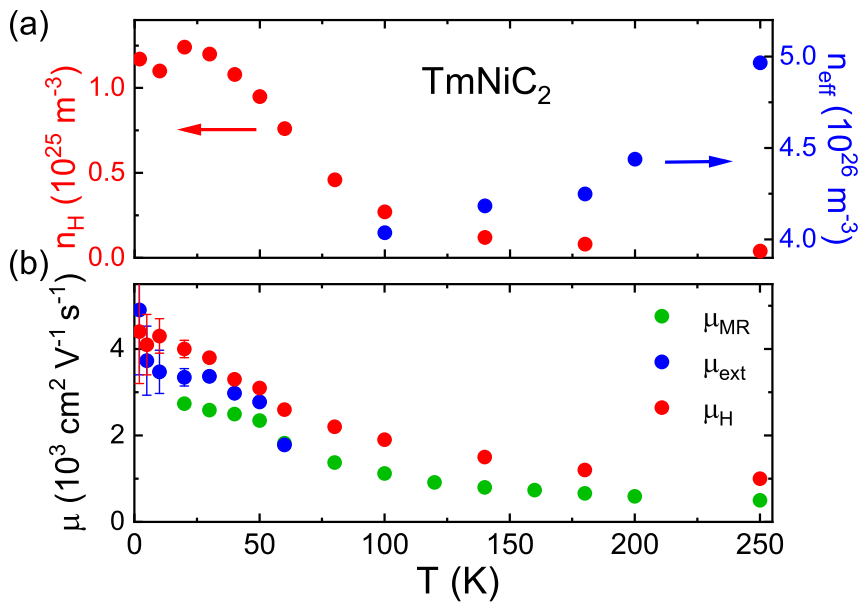

FIG. 3. Thermal dependence of concentrations (a): majority carriers $n_{\text {eff }}$ estimated from the high limit of $\rho_{y x}\left(\mu_{0} H\right)$, right scale, minority carriers $n_{H}$ from fits with Eq. (4), left scale; and mobilities (b) of the minority band of, calculated by various methods: $\mu_{\mathrm{MR}}$ from Eq. (5), $\mu_{\mathrm{ext}}$ from extremum $\sigma_{x y}\left(\mu_{0} H\right)$ and $\mu_{H}$ from fits with Eq. (4). 
the majority carriers $n_{\text {eff }}$, estimated from the high field limit of Hall resistivity as $n_{\text {eff }}=\left(\rho_{y x} /\left[\mu_{0} H\right]\right)^{-1}$ [63]. This effect is associated to the nesting of the Fermi surface in the CDW state. The minority carriers likely originate from the small pockets remaining in the FS after strong, yet imperfect nesting characteristic for quasi-2D metals. The direction of the change of the overall Hall signal, which shifts from negative values closer to zero suggests their hole origin, yet one cannot completely exclude the presence of an electron pocket. The mobility of these carriers, shown in Fig. 3(b), increases as temperature is lowered, and attains the maximum value of approx $4-5 \times 10^{3} \mathrm{~cm}^{2} \mathrm{~V}^{-1} \mathrm{~s}^{-1}$ (depending on the estimation method) without a tendency to decrease even in the AFM or field induced MM or FM states. The obtained values of $\mu_{\mathrm{ext}}, \mu_{H}$ and $\mu_{\mathrm{MR}}$ are in a qualitative agreement with each other, with the maximum 20\%-25\% difference between them indicating that each of these methods serves as a good estimate. The mobility we find, as well as the character of the evolution in the electronic transport properties in $\mathrm{TmNiC}_{2}$ is on par with the features found in the paramagnetic $\mathrm{YNiC}_{2}$ where the CDW state was not interrupted by magnetism. It should also be noted that, even considering the most conservative estimate of $4 \times 10^{3} \mathrm{~cm}^{2} \mathrm{~V}^{-1} \mathrm{~s}^{-1}$ at $2 \mathrm{~K}$, this result parallels the scale of mobility seen in magnetic materials hosting Dirac electrons $[60,64,65]$ or Weyl nodes [66]. Large $\mu$ has recently been reported also in magnetic state of $\mathrm{GdTe}_{3}$ [8], yet the role played by $\mathrm{CDW}$ was found not to be significant. In $\mathrm{TmNiC}_{2}$, the electronic mobility, similarly to high field MR shows a smooth and continuous temperature dependence, scarcely affected by magnetic transitions. This leads to the conclusion that the high mobility pockets, intimately related to FS nesting, remain intact.

In summary, we have studied the transport and magnetotransport properties of $\mathrm{TmNiC}_{2}$. We find that the Peierls transition causes a large condensation of the majority carriers (electrons), concomitant with opening of small Fermi surface pockets containing high mobility carriers (predominantly holes). This leads to the emergence of high magnetoresistance, reaching $440 \%$ at $T=2 \mathrm{~K}$, remaining linear with magnetic field and showing no signatures of saturation up to $\mu_{0} H=9 \mathrm{~T}$. The high mobility determined separately by three different approaches attains $4 \times 10^{3} \mathrm{~cm}^{2} \mathrm{~V}^{-1} \mathrm{~s}^{-1}$ in the ferromagnetic state, paralleling the scale of $\mu$ observed in a number of magnets with topological features in the electronic structure. In contrast to other $R \mathrm{NiC}_{2}$ materials in which the CDW is suppressed by ferromagnetism, in transport properties of $\mathrm{TmNiC}_{2}$ we observe no signatures of the deterioration of nesting properties. The field induced transitions are accompanied neither by a strong negative MR nor by rapid upturn in Hall resistivity due to the release of previously condensed carriers. Contrarily, the persistence of large positive MR, whose origin remains unchanged across the FM crossover as evidenced by Kohler's scaling strongly suggests the coexistence of CDW and ferromagnetism. Therefore, the Fermi surface reconstruction due to the charge density wave appears to be an alternative precursor to maintain the high carrier mobility in the magnetically ordered state, when there is no destructive interaction between these two entities.

Authors gratefully acknowledge the financial support from National Science Centre (Poland), from Grants No. DEC-2018/28/T/ST3/00164 (M. R) and 2015/19/B/ ST3/03127 (T. K.)

[1] A. Fert and F. N. V. Dau, C.R. Phys. 20, 817 (2019).

[2] V. K. Joshi, Eng. Sci. Technol. Int. J. 19, 1503 (2016).

[3] T. Chen, W. Liu, F. Zheng, M. Gao, X. Pan, G. van der Laan, X. Wang, Q. Zhang, F. Song, B. Wang, B. Wang, Y. Xu, G. Wang, and R. Zhang, Adv. Mater. 27, 4823 (2015).

[4] J. Liu, J. Hu, H. Cao, Y. Zhu, A. Chuang, D. Graf, D. J. Adams, S. M. A. Radmanesh, L. Spinu, I. Chiorescu, and Z. Mao, Sci. Rep. 6, 30525 (2016).

[5] J. Y. Liu, J. Hu, Q. Zhang, D. Graf, H. B. Cao, S. M. A. Radmanesh, D. J. Adams, Y. L. Zhu, G. F. Cheng, X. Liu, W. A. Phelan, J. Wei, M. Jaime, F. Balakirev, D. A. Tennant, J. F. DiTusa, I. Chiorescu, L. Spinu, and Z. Q. Mao, Nat. Mater. 16, 905 (2017).

[6] S. Huang, J. Kim, W. A. Shelton, E. W. Plummer, and R. Jin, Proc. Natl. Acad. Sci. U.S.A. 114, 6256 (2017).

[7] H. Li, S. Liu, C. Liu, J. Zhang, Y. Xu, R. Yu, Y. Wu, Y. Zhang, and S. Fan, Phys. Chem. Chem. Phys. 22, 556 (2020).

[8] S. Lei, J. Lin, Y. Jia, M. Gray, A. Topp, G. Farahi, S. Klemenz, T. Gao, F. Rodolakis, J. L. McChesney, C. R. Ast, A. Yazdani, K. S. Burch, S. Wu, N. P. Ong, and L. M. Schoop, Sci. Adv. 6, eaay6407 (2020).

[9] J. M. D. Coey, Magnetism and Magnetic Materials (Cambridge University Press, Cambridge, England, 2010).

[10] P. Monceau, Adv. Phys. 61, 325 (2012).

[11] G. Grüner, Density Waves in Solids, Advanced Book Program: Addison-Wesley (Perseus Books Group, Cambridge, 2000).

[12] A. Rötger, J. Lehmann, C. Schlenker, J. Dumas, J. Marcus, Z. S. Teweldemedhin, and M. Greenblatt, Europhys. Lett. 25, 23 (1994).

[13] J.-S. Rhyee, K. H. Lee, S. M. Lee, E. Cho, S. I. Kim, E. Lee, Y. S. Kwon, J. H. Shim, and G. Kotliar, Nature (London) 459, 965 (2009).

[14] H. Cho, J. H. Yun, J. H. Kim, S. Y. Back, H. S. Lee, S. J. Kim, S. Byeon, H. Jin, and J.-S. Rhyee, ACS Appl. Mater. Interfaces 12, 925 (2020).

[15] S. Kawasaki, Z. Li, M. Kitahashi, C. T. Lin, P. L. Kuhns, A. P. Reyes, and G.-q. Zheng, Nat. Commun. 8, 1267 (2017).

[16] A. O. Fumega, M. Gobbi, P. Dreher, W. Wan, C. GonzálezOrellana, M. Peña-Díaz, C. Rogero, J. Herrero-Martín, P. Gargiani, M. Ilyn, M. M. Ugeda, V. Pardo, and S. BlancoCanosa, J. Phys. Chem. C 123, 27802 (2019).

[17] Y.-G. Kang, S.-W. Kim, and J.-H. Cho, Phys. Rev. B 96, 235416 (2017). 
[18] S. Ramakrishnan, A. Schönleber, T. Rekis, N. van Well, L. Noohinejad, S. van Smaalen, M. Tolkiehn, C. Paulmann, B. Bag, A. Thamizhavel, D. Pal, and S. Ramakrishnan, Phys. Rev. B 101, 060101(R) (2020).

[19] S. Shimomura, C. Hayashi, N. Hanasaki, K. Ohnuma, Y. Kobayashi, H. Nakao, M. Mizumaki, and H. Onodera, Phys. Rev. B 93, 165108 (2016).

[20] N. Yamamoto, R. Kondo, H. Maeda, and Y. Nogami, J. Phys. Soc. Jpn. 82, 123701 (2013).

[21] A. Wölfel, L. Li, S. Shimomura, H. Onodera, and S. van Smaalen, Phys. Rev. B 82, 054120 (2010).

[22] H. Maeda, R. Kondo, and Y. Nogami, Phys. Rev. B 100, 104107 (2019).

[23] J. Yakinthos, P. Kotsanidis, W. Schäfer, and G. Will, J. Magn. Magn. Mater. 89, 299 (1990).

[24] N. Hanasaki, K. Mikami, S. Torigoe, Y. Nogami, S. Shimomura, M. Kosaka, and H. Onodera, J. Phys. Conf. Ser. 320, 012072 (2011).

[25] S. Shimomura, N. Hanasaki, H. Nakao, and H. Onodera, J. Phys. Soc. Jpn. Conf. Proc. 30, 011081 (2020).

[26] N. Hanasaki, S. Shimomura, K. Mikami, Y. Nogami, H. Nakao, and H. Onodera, Phys. Rev. B 95, 085103 (2017).

[27] M. Roman, T. Klimczuk, and K. K. Kolincio, Phys. Rev. B 98, 035136 (2018).

[28] M. Roman, L. Litzbarski, T. Klimczuk, and K. K. Kolincio, Phys. Rev. B 99, 245152 (2019).

[29] M. A. Ruderman and C. Kittel, Phys. Rev. 96, 99 (1954).

[30] T. Kasuya, Prog. Theor. Phys. 16, 45 (1956).

[31] K. Yosida, Phys. Rev. 106, 893 (1957).

[32] K. K. Kolincio, K. Górnicka, M. J. Winiarski, J. Strychalska-Nowak, and T. Klimczuk, Phys. Rev. B 94, 195149 (2016).

[33] K. K. Kolincio, M. Roman, M. J. Winiarski, J. StrychalskaNowak, and T. Klimczuk, Phys. Rev. B 95, 235156 (2017).

[34] H. Lei, K. Wang, and C. Petrovic, J. Phys. Condens. Matter 29, 075602 (2017).

[35] S. Shimomura, C. Hayashi, G. Asaka, N. Wakabayashi, M. Mizumaki, and H. Onodera, Phys. Rev. Lett. 102, 076404 (2009).

[36] N. Hanasaki, Y. Nogami, M. Kakinuma, S. Shimomura, M. Kosaka, and H. Onodera, Phys. Rev. B 85, 092402 (2012).

[37] K. K. Kolincio, M. Roman, and T. Klimczuk, Phys. Rev. B 99, 205127 (2019).

[38] M. Roman, J. Strychalska-Nowak, T. Klimczuk, and K. K. Kolincio, Phys. Rev. B 97, 041103(R) (2018).

[39] Y. Koshikawa, H. Onodera, M. Kosaka, H. Yamauchi, M. Ohashi, and Y. Yamaguchi, J. Magn. Magn. Mater. 173, 72 (1997).

[40] P. Kotsanidis, J. Yakinthos, and E. Gamari-Seale, J. LessCommon Met. 152, 287 (1989).

[41] W. Schäfer, G. Will, J. Yakinthos, and P. Kotsanidis, J. Alloys Compd. 180, 251 (1992).

[42] See the Supplemental Material at http://link.aps.org/ supplemental/10.1103/PhysRevLett.125.176601 for the details of structural analysis and the computational methods, which includes Refs. [9,23,24,32-34,40,41,43].
[43] H. Onodera, Y. Koshikawa, M. Kosaka, M. Ohashi, H. Yamauchi, and Y. Yamaguchi, J. Magn. Magn. Mater. 182, 161 (1998).

[44] M. M. Parish and P. B. Littlewood, Nature (London) 426, 162 (2003).

[45] M. M. Parish and P. B. Littlewood, Phys. Rev. B 72, 094417 (2005).

[46] A. A. Abrikosov, J. Exp. Theor. Phys. 29, 746(1969), http:// www.jetp.ac.ru/cgi-bin/e/index/e/29/4/p746?a=list.

[47] A. A. Abrikosov, Phys. Rev. B 58, 2788 (1998).

[48] A. A. Abrikosov, Europhys. Lett. 49, 789 (2000).

[49] A. A. Sinchenko, P. D. Grigoriev, P. Lejay, and P. Monceau, Phys. Rev. B 96, 245129 (2017).

[50] A. V. Frolov, A. P. Orlov, P. D. Grigoriev, V. N. Zverev, A. A. Sinchenko, and P. Monceau, JETP Lett. 107, 488 (2018).

[51] Y. Feng, Y. Wang, D. M. Silevitch, J.-Q. Yan, R. Kobayashi, M. Hedo, T. Nakama, Y. Onuki, A. V. Suslov, B. Mihaila, P. B. Littlewood, and T. F. Rosenbaum, Proc. Natl. Acad. Sci. U.S.A. 116, 11201 (2019).

[52] I. M. Lifshitz, M. I. Azbel, and M. I. Kaganov, J. Exp. Theor. Phys. 4, 41 (1957), http://www.jetp.ac.ru/cgi-bin/e/ index/e/4/1/p41? a=list.

[53] S. N. Zhang, Q. S. Wu, Y. Liu, and O. V. Yazyev, Phys. Rev. B 99, 035142 (2019).

[54] M. Kohler, Ann. Phys. (Berlin) 424, 211 (1938).

[55] A. Pippard, Magnetoresistance in Metals, Cambridge Studies in Low Temperature Physics (Cambridge University Press, Cambridge, England, 2009).

[56] N. Nagaosa, J. Sinova, S. Onoda, A. H. MacDonald, and N. P. Ong, Rev. Mod. Phys. 82, 1539 (2010).

[57] T. Liang, Q. Gibson, M. N. Ali, M. Liu, R. J. Cava, and N. P. Ong, Nat. Mater. 14, 280 (2015).

[58] J. Fujioka, R. Yamada, M. Kawamura, S. Sakai, M. Hirayama, R. Arita, T. Okawa, D. Hashizume, M. Hoshino, and Y. Tokura, Nat. Commun. 10, 362 (2019).

[59] A. F. May, M. A. McGuire, and B. C. Sales, Phys. Rev. B 90, 075109 (2014).

[60] Y.-Y. Wang, S. Xu, L.-L. Sun, and T.-L. Xia, Phys. Rev. Mater. 2, 021201 (2018).

[61] H. Takahashi, R. Okazaki, Y. Yasui, and I. Terasaki, Phys. Rev. B 84, 205215 (2011).

[62] S. Ishiwata, Y. Shiomi, J. S. Lee, M. S. Bahramy, T. Suzuki, M. Uchida, R. Arita, Y. Taguchi, and Y. Tokura, Nat. Mater. 12, 512 (2013).

[63] C. M. Hurd, The Hall Effect in Metals and Alloys (Springer, Berlin, 1972).

[64] J. Park, G. Lee, F. Wolff-Fabris, Y. Y. Koh, M. J. Eom, Y. K. Kim, M. A. Farhan, Y. J. Jo, C. Kim, J. H. Shim, and J. S. Kim, Phys. Rev. Lett. 107, 126402 (2011).

[65] H. Masuda, H. Sakai, M. Tokunaga, Y. Yamasaki, A. Miyake, J. Shiogai, S. Nakamura, S. Awaji, A. Tsukazaki, H. Nakao, Y. Murakami, T.-h. Arima, Y. Tokura, and S. Ishiwata, Sci. Adv. 2, e1501117 (2016).

[66] M. Hirschberger, S. Kushwaha, Z. Wang, Q. Gibson, S. Liang, C. Belvin, B. A. Bernevig, R. J. Cava, and N. P. Ong, Nat. Mater. 15, 1161 (2016). 